Tuesday, 2003 April 15, 23:26:13

Sunday, 2000 July 16, 19:50:10

\title{
Color Rendering: Asking the Question
}

James A. Worthey, 11 Rye Court, Gaithersburg, Maryland, 20878-1901, USA.

Abstract

Two white lights may have the same chromaticity, and yet when used to light an array of objects may differ in their ability to reveal colors. For example, any white light can be matched by a mixture of two narrow-band lights, a yellow plus a blue. In this extreme case, reds and greens become black or brown and the red-green dimension is lost. At the other extreme, a light with three narrow bands, at the proper wavelengths, can brighten reds and greens and increase redgreen contrast, relative to a broad-band light such as daylight. Many commercial lights tend to dull reds and greens, relative to broad-band sources, a central reason that color rendering is a practical concern. A telling example is neodymium glass, a yellow-absorbing filter that is sometimes used to improve color rendering. This article seeks to bring these ideas to life through detailed graphical examples.

Keywords: ASTM, Color Rendering, Colorimetry, Fluorescent Lighting, Illuminants, Lighting, Linear Model, Matrix R, Neodymium Glass, Opponent Color, Prime Colors, Standards.

\section{Introduction: rendering by light sources}

The everyday function of vision is to gather information about non-luminous objects. One or a few luminous objects must be present, as sources for light, which is then reflected or absorbed by non-luminous things according to their optical properties and those of the source(s). The reflected light carries information in the form of black-white and color contrasts. This process is summarized in one word by saying that the light renders the object properties. An object feature may be better rendered by one light than by another.

For instance, if the object is a clear wine glass, it has no intrinsic areas of light, dark, or color. It reflects and refracts the contrasts of its environment. Of particular interest are highlights, which are images of light source(s) reflected in the glass surface. Highlights (and all other surface reflections) will have a luminance on the order of $4 \%$ of the luminance of the thing reflected ${ }^{1}$. A compact high-luminance source, such as a filament lamp, will render the glass as a sparkling high-contrast object, while in a featureless environment such as an integrating sphere, highlights as such will be absent and the glass will be almost invisible.

People so trust the eye's ability to decode available cues, that everyday speech ignores the role of the light: "An apple is shiny and red; a glass sparkles." In such phrasing, there is an implicit trust that the light is doing its job. A lighting system should earn this trust, rendering objects with revealing black-white and color contrasts. In fact, it is scarcely an exaggeration to say that people must trust the light, because common language and assumptions do not permit rendering issues to be expressed. Suppose that a room has a number of electric lamps, of whatever technology, visible as one enters the room. If those lamps are not closely matched in 
chromaticity, people will notice and indeed complain. This is a well-known issue that has motivated research, standards development, and so forth. The color variation may have little effect on vision of objects, but it is something that a visitor can quickly notice and describe.

On the other hand, suppose there is a second similar room whose lights are held to a tight chromaticity tolerance. The lights are a pleasing white color when viewed directly. These hypothetical lights have only one flaw. When they are used to illuminate objects in the room, all red and green objects show up as black or brown. An example below will show how this can be done. A visitor who steps into the room will experience the problem, but his senses and his vocabulary will fail him in trying to explain what is happening. He may say that the room is "dark," which is true in a sense, even if not true to an illuminance meter. These lights create a much worse problem than do the mixed-color lights, but the visitor is less able to describe where the lights have fallen short.

Any discussion of rendering is beset with this issue. Many common lighting systems render objects with black-white and color contrasts that are less than they could be. The lighting user may well sense that something is wrong, but her senses and the language fail her if she seeks to explain the problem. The problem is not vague or mysterious; it is physical and measurable; it is just hard to verbalize. In part, this article is about finding the right words to describe the rendering of colors by everyday lights.

\section{Approach}

When reference is made to "black-white and color contrasts," the reader may wish to picture a diverse collection of objects, with their color differences evaluated in a nonlinear color space such as CIELAB. This vision captures the key idea of color differences among objects seen under a common light source. However, in this article and the next one, the transformation to a nonlinear color space will not be done. My approach is that of a physicist or a lighting inventor, focused on the physical process by which color stimuli are created. Insights will be gained that have everything to do with basic color mixing and nothing to do with color-difference metrics.

\section{Goal}

This article is concerned primarily with color rendering, studied by analysis and examples. Do all lights earn our trust by revealing objects in their innate lightness and chromaticity? Can a light lose object chroma, or exaggerate it? In a second article $^{2}$, a new calculation method for color rendering will be presented. Among other things, the new method can be used to compare light sources, potentially replacing the established color rendering algorithm ${ }^{3}$.

The new method is based on a few clearly stated assumptions, and gives numerical answers to questions such as those just stated. It is easy to express as a computer program, since the formulas involve mainly simple matrix operations. Rooted in orderly science, the formulas can potentially be linked to discussions of computer graphics, machine vision and color constancy, where similar methods have been used.

The new method uses the idea of opponent colors, and in this is related to one previously published ${ }^{4}$. Echoing a suggestion of Thornton ${ }^{5,6}$, Xu published a simple theory of color 
rendering, based on the notion that some lights shrink the gamut of available colors ${ }^{7}$. These prior theories have had limited effect on the public discussion of lighting, perhaps because they answered a question that readers were not asking. The purpose of this article, then, is to ask the questions, to raise the reader's awareness of color rendering. This topic deserves greater scientific attention, and more respect as a practical concern in lighting.

\section{Color Rendering}

Imagine a brightly printed silk scarf spread out on a table, and on it a bowl of fruits and vegetables. These objects have differing spectral reflectances, meaning in effect that they have various innate colors. If green and red bell peppers are among the objects, under suitable lighting, they should appear quite dissimilar and be unmistakably green and red. The printed scarf was intended by its maker to be bright, meaning that the colors are intended to present high chroma and lightness.

Can a light source diminish color contrast? If our collection of objects is lighted exclusively by a low-pressure sodium vapor light, which is essentially a monochromatic source at $589 \mathrm{~nm}$, all color contrast is lost. Areas of saturated red, green, or blue become black. The potential for a light to reveal or conceal colors represents a $100 \%$ variation, from colorful and faithful to the expected hues, to no color at all.

Ives $^{8}$ and Thornton and Chen ${ }^{6}$ have called attention to another type of source that sharply reduces color contrast, that is the two-narrow-bands light ${ }^{9}$. A white such as $4002 \mathrm{~K}$ blackbody radiation $[(\mathrm{x}, \mathrm{y})=(0.3804,0.3767)]$ can be metameric to a mixture of a narrow band in the blue $(446 \mathrm{~nm})$, plus a narrow band in the yellow $(574 \mathrm{~nm})$. Under this light, all the object chromaticities fall along a straight line in the chromaticity diagram. A range of colors may be seen, from light to dark and blue to white to yellow, but all reds and greens are lost, meaning that red-green contrast is missing, Fig. $1^{9}$.

Lights such as daylight and blackbody radiation spread their radiant power broadly through the domain of visible wavelengths. Between such spectrally broad sources and the two-narrowbands light is a range of possibilities in which the two bands are broadened, but the spectrum rises in the yellow and dips in the red and the green. Logic says that such intermediate lights will have an intermediate effect: systematically dulling reds and greens, and shrinking red-green contrasts ${ }^{4}$. Many common vapor-discharge lamps have this color-losing quality.

In Fig. 2, the spectra of four lights are normalized to the same illuminance. They are also chosen to have similar chromaticities $(x, y)$ in the CIE system. Three are broad-band sources: a daylight computed by the method of Judd, MacAdam, and Wyszecki ${ }^{10}$; blackbody radiation at $4002 \mathrm{~K}$; and a commercial filtered filament lamp intended for color viewing ${ }^{11}$. The fourth source is not broad-band: a cool white fluorescent lamp ${ }^{12}$. The fluorescent lamp's spectral power distribution (SPD) rises above the others at certain bluish and yellowish wavelengths, while falling below them in the red and green. This is not a unique example, but similar to comparisons that could be generated for other fluorescent lamp types, and other vapor discharge lights ${ }^{13}$. 


\section{Color Rendering in the Laboratory}

If a 2-band light loses object color contrast, what might a 3-band light do? McCann, McKee, and Taylor reported a color constancy experiment, under the title of "Quantitative studies in retinex theory." ${ }^{\prime 14}$ In order to create a test light that could be adjusted in chromaticity as well as intensity, the authors followed a method of Edwin Land ${ }^{15}$. As a light source, three projectors were used, each containing a narrow-band filter. The narrow bands peaked at 630,530, and $450 \mathrm{~nm}$, in the red, the green, and the blue, bandwidth $10 \mathrm{~nm}$ at half-height. The intensity of each projector was separately adjustable, but the beams were superimposed to illuminate an array of test papers.

Not only did three projectors jointly illuminate the test papers, but a second triplet of projectors with narrow-band filters was used to illuminate the comparison papers, as the authors explain:
"Our choice for the desired 'catalogue' of color sensations was the Munsell Book of Color (Matte Finish Collection). We then had to select an appropriate illuminant. We could have used a broad-band illuminant such as Illuminant $\mathrm{C}$, used in the original selection and definition of Munsell papers; instead we decided to use the three narrow-band illuminants used to illuminate the Mondrian. The reason for this choice is that the papers in the Munsell Book viewed in Illuminant $C$ do not appear as saturated as the same Munsell papers viewed in three narrow-band illuminants." ${ }^{\prime 4}$ (Boldface added.)

To review, these authors set out to do a color constancy experiment. Constancy is often stated in general terms to imply that if the SPD of the light is changed, the visual system will counteract that change so that objects give invariant color sensations. McCann et al. say as much. Yet in this case, if we consider the lighting change (Light C) $\rightarrow$ (3-band Light), the effects of the change were significant and perhaps of a type that the visual system would not counteract. In any event, they were of a type that the authors did not wish to study. McCann et al. found a degree of constancy in an experiment where the independent variable was the light source's color, both chromaticity and intensity. A decision was made to exclude a color rendering phenomenon by using 3-band lights consistently in the experiment.

Fig. 3 (of the present article) shows what a 3-band light can do. This figure is based entirely on detailed calculations, and not on data from the quantitative retinex article. Only the concept of the 3-band Light is preserved, with its set of wavelengths, $\{630 \mathrm{~nm}, 530 \mathrm{~nm}, 450 \mathrm{~nm}\}$. The arrows correspond to the 64 Munsell papers whose spectral reflectance factors were measured by Vrhel, Gershon, and Iwan. ${ }^{16}$ Each arrow tail is a paper's chromaticity under Light C, calculated from the spectral power distribution (SPD) of Light $C$, and the spectral reflectance. An idealized 3-band light was generated by assuming a band only $1 \mathrm{~nm}$ wide at each center wavelength, and adjusting the intensity of the bands to make the 3-band light metameric to Light C. (Adjusting the 3-band light is a small matrix-algebra exercise.) Then each arrowhead shows the chromaticity of the paper under the 3-band light. The three narrow bands themselves are indicated by diamond shapes, $\diamond$.

In Fig. 3, the passing observation of McCann et al. is clearly true: "the papers in the Munsell Book viewed in Illuminant $C$ do not appear as saturated as the same Munsell papers 


\section{viewed in three narrow-band illuminants." 14}

\section{Digression}

Fig. 4 shows what McCann et al. were intending to work on. There were 18 Munsell papers in the test display. The solid arrows show the colorimetric shifts of the 18 papers when the 3-band light is adjusted from a neutral color (in the "gray experiment") to a bluish color (in the "yellow experiment") ${ }^{14}$. The heads of the dashed arrows show the perceived chromaticities of those papers, so that the dashed arrows are the perceptual shifts. To the extent that each dashed arrow is the vector negative of the associated solid arrow, color constancy occurred ${ }^{9,14}$. (As stated, Fig. 4 includes human responses; it also is based on certain approximations; in this article, it is the only figure to have these features. See reference 9 for details.)

\section{Moment of Reflection}

Yogi Berra said "You can see a lot just by looking." The reader is asked to pause and study Fig. 3. This is a realistic but particularly uncomplicated example because of the smooth and orderly reflectances of the Munsell papers. ${ }^{9,16,17}$ Light $\mathrm{C}$ and the Munsell papers are normal textbook constructs. The 3-band light, though unusual, is simple.

The substitution of lights causes many colors to increase in spectral purity. Both lights have the same chromaticity, marked by + . The 3-band light makes the reddish papers redder and the greenish papers greener. Suppose that, within a collage, a reddish paper lies next to a greenish one, or even a neutral or yellowish one. Then the effect of (Light C) $\rightarrow(3$-band Light) is to increase color contrast.

\section{Video}

Under the 3-band light, any object will reflect a mixture of the 3 narrow bands, according to its reflectance at the 3 wavelengths. This is similar to the combining of phosphor primaries on a video display, with the difference that a video screen radiates a mixture of the phosphor radiances, whether they are narrow-band or not. The chromaticities of all objects under that light lie within the triangle determined by the chromaticities of the narrow bands. In Fig. 5, the solid triangle is based on the phosphor primaries of NTSC video ${ }^{18}$. The triangle of long dashes is based on the narrow bands of McCann et $a l^{14}$. The triangle of short dashes is based on the set of narrow bands $\{450 \mathrm{~nm}, 540 \mathrm{~nm}, 610 \mathrm{~nm}\}$, Thornton's "prime colors ${ }^{19}$."

To the designers of video tubes and television systems, it is clear that they control the rendering of colors. Color does not appear in the display until a system is established to detect and transmit color differences, and three phosphors are chosen to give a large triangular chromaticity gamut in the final display ${ }^{18}$. The purpose of the whole complicated system is to present black-white and color contrasts to humans.

Fig. 5 emphasizes the similarity of lighting to television. The choice of wavelengths for the three-band light determines a chromaticity gamut just as the choice of video phosphors does. The availability of color information to the lighting user is under engineering control, just as it is for the television viewer. Television diverges from lighting in this: within the television system are controls by which color contrast may be adjusted in two dimensions. The red-green contrast 
control, whose effects are akin to the red-green contrast effects in Fig. 3, is called "color," while the blue-yellow control is referred to as "tint."

Modern television sets may no longer have prominent color and tint knobs, but the two dimensions of color contrast still exist for the studio technician and for the designer of television sets. In the case of lighting, the knobs never existed, but the control always exists. The spectral power distribution of the light determines the size and shape of the color gamut, and the rendering of specific object colors within that gamut. As suggested by the examples of Figs. 1 and 3 , and by previous work ${ }^{4}$, the independent dimensions of red-green and blue-yellow contrast are as appropriate to lighting as to television.

As stated, television engineers must and do acknowledge that color contrast is the product—or at least a major component of what they must produce. In a different regime, visual artists such as clothing designers seek to control color, as do the chemists who supply dyes and paints. In the realm of lighting, the supposed technical experts do not acknowledge that color contrasts and black-white contrasts are the product.

Readers who are highly trained may object that the comparison of lighting and television is inexact, because the signals in a TV exist on a few distinct wires, whereas a light shines on $N$ objects, and it then operates within the $3 N$ calculations of the object tristimulus values. To this, there are two answers:

1. Indeed the comparison is not exact. One may study Hunt's The reproduction of colour in photography, printing and television ${ }^{18}$ to see that the television's additive display also differs from the subtractive color mixing in photography and from color printing where both additive and subtractive mixing operate. Nonetheless, the three reproduction technologies have been developed with similar goals of stimulating color vision. It does appear that color rendering by lights has more similarity to television than to, say, printing.

2. It is incorrect to think that because some function operates within a group of similar integrals (or sums), that its effects cannot be separated out. The separation can be done by a well-known approximation method. Approximation is just what one wants, to express the light's main effects in a few numbers, while gracefully losing some detail. This method is of course the "new method" of the later article.

\section{Prime Colors}

Fig. 3 shows that a certain 3-band light renders objects in high color contrast. This raises deeper questions. What 3 wavelengths are most effective at stimulating normal color vision? MacAdam studied this by finding those wavelengths for which the least power balances one watt of the complement in mixing to make a white ${ }^{20}$. He found maxima near 450, 540, and $610 \mathrm{~nm}$ and minima near 500 and $580 \mathrm{~nm}$. Thornton and others found similar results when the problem was posed in other ways ${ }^{20,21}$. Thornton calls the set $\{450,540,610 \mathrm{~nm}\}$ the prime colors. He also identifies 500 and $580 \mathrm{~nm}$ as least effective for stimulating color vision.

Rather than to review Thornton's work, it may be interesting to make some heuristic observations as to how the prime colors arise. In Fig. 6 is a version of human cone sensitivities,

$\{\bar{b}, \bar{g}, \bar{r}\}$, generated as linear combinations of the usual CIE color-matching functions ${ }^{2}$. Now 
suppose that one is to choose 3 wavelengths for a 3 -band lamp. Since $\bar{b}$ stands apart from the other functions, either you stimulate it or you don't; one narrow band should be put near the peak of $\bar{b}$. The other functions, $\bar{g}$ and $\bar{r}$ greatly overlap. Putting the other two narrow bands at the peak of $\bar{g}$ at $543 \mathrm{~nm}$, and the peak of $\bar{r}$ at $566 \mathrm{~nm}$ will stimulate those cones, but will give a narrow gamut with little red-green range. (The red primary would be yellow! See Fig. 5.) One wants to stimulate $\bar{g}$ more than $\bar{r}$, or the reverse. A function that expresses such differential stimulation is a red-green opponent color function ${ }^{2}, \bar{t}$, with local extrema at about 540 and $610 \mathrm{~nm}$, Fig. 7. (The actual negative and positive peaks of $\bar{t}$ in Fig. 7 are at 529 and 611 nm. )

It might seem that a fourth prime color would exist for the yellow peak (at $567 \mathrm{~nm}$ ) of the blueyellow opponent function, but that is not so. Stimulating $\bar{g}$ and $\bar{r}$ equally, a narrow band yellow light would desaturate reds and greens. Since the 540 and $610 \mathrm{~nm}$ bands give yellow when mixed, actual yellow light is not needed. Yellow object colors tend to have "minus blue" spectral reflectances, absorbing blue light while reflecting all wavelengths above, say, $500 \mathrm{~nm}^{22}$. See Fig. 8.

To put it another way, suppose that one had the 3-band lamp of McCann et al. ${ }^{14}$, and then a 4th projector were added to supply a narrow band of yellow light at $570 \mathrm{~nm}$. If the power is turned up on the yellow, then the red and green bands must be dimmed to keep the mixture's chromaticity constant, and one is moving in the direction of the 2-band light of Fig. 1.

In Fig. 5, one sees that the "prime colors" give a large triangular gamut, but not the largest possible. Blindly enlarging the triangle would mean moving each primary into a region of diminishing receptor sensitivity, Fig 6 . The prime colors are not intended to maximize the gamut, but to "deliver the goods" of chromatic color by acting strongly in mixtures.

Fig. 9 shows chromaticity shifts of 36 Munsell papers as they are illuminated by 4 lights in succession. Each paper leads to a chain of 3 arrows. The papers are a subset of those measured by $\operatorname{Vrhel}^{16}$, chosen to minimize overlap of arrows. All the neutral papers were omitted. The lights are:

1. A cool white fluorescent lamp ${ }^{23},(x, y)=(0.3786,0.3906)$.

2. JMW daylight ${ }^{10},(x, y)=(0.3786,0.3906)$. This chromaticity is actually out of the normal domain by 0.001 in the $+y$ direction.

3. Ultralume $4100 \mathrm{~K}^{24}$, a fluorescent lamp that was designed by Thornton to approximate the ideal of a prime color light. This lamp was once in commercial production, but is no longer available. $(x, y)=(0.3749,0.3889)$.

4. An idealized prime color source with narrow bands (width $=1 \mathrm{~nm}$ ) at 450, 540, and $610 \mathrm{~nm} .(x, y)=(0.3786,0.3906)$ These wavelengths are indicated on the spectrum locus, with diamond shapes, $\diamond$.

Notice that 3 of the lights match in chromaticity; the daylight and idealized prime color light were adjusted to make this happen. The first transition, cool white to daylight, shows that the traditional cool white diminishes red-green contrasts relative to daylight. This was discussed in relation to Fig. 2. The next transition, daylight to Ultralume $4100 \mathrm{~K}$, shows that a practical fluorescent lamp can increase red-green contrasts beyond those that the objects would have in 
daylight, as Thornton intended to do. The final transition, Ultralume $4100 \mathrm{~K}$ to ideal prime color, increases the spectral purity of reds and greens a bit more. The SPDs of the 4 lights are graphed in Fig. 10.

\section{Further Moment of Reflection}

Fig. 9, along with Figs. 1 and 3, illustrates a central point: color contrast is under engineering control, particularly red-green contrast. If 4 lights were chosen at random, this would still be true, but the pattern of color shifts would defy simple interpretation. This "simple" example is only possible because of

1. The daylight model of Judd, MacAdam and Wyszecki.

2. Thornton's prime color ideal.

3. Thornton's practical lamp design.

4. The Munsell Company's efforts in realizing the Munsell renotation with orderly progressions of pigment mixtures, and therefore of spectral reflectances.

5. The efforts of Vrhel et al. ${ }^{16}$ in measuring the spectral reflectances and making them available.

Three lights share the same chromaticity, while the fourth chromaticity differs only by $\Delta(x, y)=$ $(-0.0037,-0.0017)$. A chromaticity shift in the light would cause most object chromaticities to shift in similar directions, the sort of thing that is the physical basis for "color constancy" experiments.

Figs. 1, 3, and 9 reveal a shortcoming of the traditional color-rendering calculation ${ }^{3}$. Color contrast effects have a direction, an algebraic sign. If daylight is taken as a starting point, then reds and greens can become more saturated or less saturated under other lights. The CIE method $^{3}$ looks only at the magnitude of shifts and ignores their direction.

\section{Matrix R}

The central idea of "prime colors" is that 3 regions of the spectrum stand out in their ability to affect mixtures or counteract complementary wavelengths. This interesting and practical fact lurks within a set of color matching data (such as those for the CIE's 1931 observer), awaiting our notice. It is equally present whether color matching data take shape as the receptor sensitivities of Fig. 6, the opponent functions of Fig. 7, or the utterly familiar $\bar{x}, \bar{y}$, and $\bar{z}$. (The functions of Figs. 6 and 7 were calculated by adding and subtracting the members of $\{\bar{x}, \bar{y}, \bar{z}\}$. $)^{2}$

Cohen and Kappauf, concerned with invariant things that lie hidden within the data of color matching, discovered the projector matrix that they called Matrix $\mathbf{R}_{-}^{25}$ Let a matrix $A$ be defined

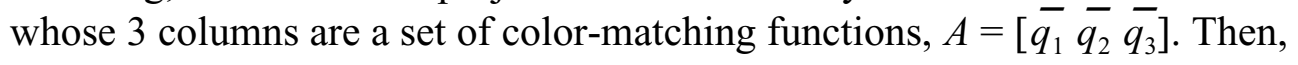

$$
\mathbf{R}=A\left(A^{\prime} A\right)^{-1} A^{\prime},
$$

where the prime, ', indicates matrix transpose. Now let the SPD of a light be represented by a 
column matrix $N$. The matrix product $R N$ will be the fundamental metamer of $N$, meaning that unique metamer which is a linear combination of color-matching functions ${ }^{25}$. Formally,

$$
N^{*}=\mathbf{R} N
$$

where $N^{*}$ is the fundamental metamer of $N$.

The symmetrical matrix $\mathbf{R}$ given by formula (1) will come out exactly the same whether $\left\{\overline{q_{1}}, \overline{q_{2}}, \overline{q_{3}}\right\}$ is $\{\bar{b}, \bar{g}, \bar{r}\},\{\bar{x}, \bar{y}, \bar{z}\}$, or some other transformed set of color-matching functions. It is the essence of an invariant thing. If two lights $N_{1}$ and $N_{2}$ are metameric, $N^{*}$ is the same for both of them.

Fundamental metamers, represented as column matrices, are analogs of colors in color space. They can be added and subtracted, and their dot products taken, representing colors in a consistent way. The row index of $N$ or the row and column indices of the rather large matrix $\mathbf{R}$ count through the wavelengths of the visible spectrum, in steps of one or more nanometers. If $N$ happens to be a narrow-band light that has unit amplitude in the $j$ th wavelength band, and zero power at other wavelengths, its fundamental metamer is the $j$ th column of $\mathbf{R}$.

In short, the columns (or rows) of $\mathbf{R}$ are the fundamental metamers of the spectral colors. If $N^{*}{ }_{\mathrm{j}}$ is the $j$ th column vector, since it is a fundamental metamer,

$$
\mathbf{R} N^{*}{ }_{\mathrm{j}}=N_{\mathrm{j}}^{*} .
$$

By normal matrix multiplication, and remembering that the $j$ th row of $\mathbf{R}$ equals $N_{\mathrm{j}}^{*}$, this implies that

$$
\left(N^{*}\right)_{\mathrm{j}}=\sum_{i}\left(N^{*}\right)_{\mathrm{i}}^{2}
$$

In other words, the $j$ th element of $N^{*}$ is itself the squared vector magnitude of $N^{*}$. This squared vector magnitude is also the $j$ th diagonal element of $\mathbf{R}$.

I apologize for the tricky notation in Eq. (4). $N^{*}$ is the fundamental metamer of the $j$ th narrow wavelength band. At the same time, it is a function of wavelength, represented as a column matrix. One subscript selects the vector and the other picks an element of the vector.

The diagonal of $\mathbf{R}$ therefore represents the squared magnitude of spectral (narrow-band) lights in color space ${ }^{25}$. The magnitude in question is related only to color mixing, and not to other amplitude issues that one might think of, such as detection of dim lights. Color mixing of suprathreshold lights is precisely the aspect of vision to which color rendering is related.

In Fig. 11 are graphed the diagonal of Matrix $\mathbf{R}$ and its square root. The square root of the diagonal shows the comparative strength of the colors of the spectrum, for the CIE $2^{\circ}$ (1931) observer, in a form that incorporates no arbitrary assumptions. The 3 peaks are positioned at 
445, 536, and $604 \mathrm{~nm}$. These values are quite close to Thornton's prime colors, 450, 540, $610 \mathrm{~nm}$. In the diagonal of Matrix $\mathbf{R}$, the minima occur at 491 and $572 \mathrm{~nm}$, comparable to Thornton's least effective wavelengths at 500 and $580 \mathrm{~nm}$.

No originality is claimed for anything in this section. These are Jozef Cohen's ideas. Matrix $\mathbf{R}$ was calculated and graphed afresh, but that is quite easy, which is part of the genius in this method.

\section{Neodymium Glass}

If a wavelength-selective filter, of spectral transmittance $\pi(\lambda)$, is put over the eye, or over a light source, this is an issue of color rendering. Any method used to study color rendering by lights can be applied as well to the study of sunglasses, for instance. Putting the filter over $L_{1}$ creates $L_{2}$ $=\pi(\lambda) L_{1}$, and thus the transition $L_{1} \rightarrow L_{2}$.

In the lighting business, a long-standing niche product is an incandescent lamp with neodymium glass ${ }^{26}$. This particular filter, consisting of glass with a small percentage of $\mathrm{Nd}_{2} \mathrm{O}_{3}$, absorbs strongly within a narrow band in the yellow part of the spectrum, Fig. 12. This graph is based on measurement of a particular glass ${ }^{27}$ in its clear state, and then with $4.2 \% \mathrm{Nd}_{2} \mathrm{O}_{3}$. The transmittance functions at the $1.7 \%$ and $6.3 \%$ levels were calculated, using Beer's law to adjust the internal transmittance ${ }^{28}$. In Fig. 12, the strongest absorption (lowest transmittance) is at about $583 \mathrm{~nm}$, almost exactly the $580 \mathrm{~nm}$ wavelength given by Thornton as ineffective for stimulating color vision.

Fig. 13 uses the same 36 Munsell papers as in Fig. 9. It is now assumed that a $2900 \mathrm{~K}$ blackbody, similar to a tungsten-halogen lamp, is filtered by the glasses of Fig. 12, starting with clear glass. It appears that the filter glass increases red-green contrasts, as expected. It also causes an overall color shift.

Bouma advocates a form of neodymium glass in a 1938 article $^{22}$. He addresses the "...requirement...that the surroundings shall have a pleasant, cosy appearance, that persons shall have a healthy appearance, that certain articles appear fresh and tasteful, etc. We shall concern ourselves here with the question of whether electric light can also be improved in this respect by the introduction of a coloured envelope. ${ }^{22,}$

He then asks what wavelength is a candidate to be filtered out, and notes

"In general, absorption of a given colour is accompanied by the following two objections:

1) An object which reflects almost exclusively this colour appears too dark.

2) Objects which exhibit the colour under consideration in a less saturated form appear still less saturated. ...

"For Yellow, however, the situation is different. Highly saturated yellow occurs in nature as a rule, not because only a narrow region of the spectrum is reflected, but because red and green as well as yellow are fairly well reflected, and only blue and violet are absorbed to a large extent. ${ }^{22,}$ 
This is an early version of the prime color idea, explained intuitively in the context of finding a filter for practical use. Bouma conducted experiments with the "colour cards of Ostwald's colour atlas $^{22}$," and noted these effects of neodymium glass:

"The orange is shifted toward the red: the shift in the direction yellow to red is in general experienced as an increased 'warmth' of that colour. This shift also gives a more healthy appearance to the complexion. The green, which upon transition from daylight to incandescent light had become a somewhat dubious yellowgreen, goes back to green again under the influence of the 'Philiphane' glass. ${ }^{22}$ ",

In other words, the selective absorption of a band in the yellow gave a perceptible and desirable increase in red-green contrast.

\section{Metamerism}

Two objects may match under a light $L_{1}$, even though their reflectances differ. A lighting transition, $L_{1} \rightarrow L_{2}$, may reveal this object color metamerism, rendering the two objects in contrasting colors ${ }^{29}$. This may be seen as a color rendering issue, because $L_{1} \rightarrow L_{2}$ and then some color "looks wrong." A difference resides in the fact that object metamerism can very well be revealed if $L_{1}$ and $L_{2}$ are blackbody radiations of different temperatures ${ }^{29}$. Such a transition between broadband lights of different colors is not what gives rise to the traditional color rendering discussion. For now, we should say that the two issues are closely related, and let it rest.

\section{Summary}

Everyday vision depends on the interaction between a light source and objects in the environment. This dependency is more than an abstract issue because lights vary greatly in area ${ }^{1}$ and in spectral power distribution, SPD. The light's role may be emphasized by saying that the light renders the object. Many objects are spectrally selective, and can present colors both subtle and bold, but this depends on the light.

Familiar lights vary in chromaticity, and the change from a yellowish light to a bluish one can cause large changes in object chromaticities, Fig. 4. "Color rendering" refers especially to the differences between lights whose chromaticities are equal, or nearly equal. By the exact choice of the blue and yellow wavelengths, and by the adjustment of their intensities, the 2-band light of Fig. 1 could be set to be a chromaticity match for any phase of daylight, for instance. Yet a scene lighted by the 2-band light would lack reds and greens, its range of colors quite impoverished.

Lighting objects with 3 well-chosen narrow bands can afford true trichromatic stimulation, Figs. 3,5 , and 9. There is a certain intuitive truth here. Objects can display colors in three dimensions only if the light source supplies power in three primary colors. Lighting should honor trichromatic vision just as television does. Daylight presumably meets this requirement with its broad spectrum.

The traditional color rendering discussion evaluates color shifts without regard to their direction $^{3}$. This exaggerates the significance of the reference light, which is assumed to render certain test papers in their uniquely correct tristimulus values, with all shifts then counted as equally bad. The present emphasis on color contrast speaks to the practical reality, that many 
common artificial lights tend to desaturate reds and greens. Natural daylight affords good color contrast; it is more than an arbitrary reference.

\section{Lightness}

The CIE chromaticity diagram has been used to illustrate rendering effects. This shows the rendering of color contrasts in an intuitive way, but loses any information about lighting effects on luminous reflectance. Suppose that $L_{1}$ is the daylight phase graphed in Fig. 2 , and $L_{2}$ is the cool white light graphed in that figure. Then as $L_{1} \rightarrow L_{2}$, saturated reds and greens will lose lightness, as well as chroma. This can be inferred by examining the graphs, noting that a saturated red object reflects light mainly in a region where the cool white light lacks spectral power. This is an additional reason for objects to appear "dull" under such a light. For less saturated colors, lightness effects are less predictable.

\section{Conclusion}

Light sources control what we see, including the black-white and color contrasts of objects. The topics of color constancy, color rendering, and object color metamerism all involve a substitution of one light for another, $L_{1} \rightarrow L_{2}$, but can be distinguished. Constancy, a response of the visual system, is expected when $L_{1}$ and $L_{2}$ are different colors, Fig. 4. Object-color metamerism can also be revealed by a color shift in the light ${ }^{29}$ (though not by the lights of Fig. $4^{9}$ ). Color rendering is best understood as an issue that arises when the two lights have the same or similar chromaticities, but differ in their ability to reveal color contrasts.

If the eye has 3 cone types, and therefore 3 primaries are needed to span color space, as symbolized in Fig. 5, how is it that a 2-band light can be metameric to a broad-band one, in Fig. 1? This is due to the overlap of the red and green cone spectral sensitivities, Fig. 6 . The two cone species are both stimulated by the yellow light. Opponent colors, prime colors, and the diagonal of Matrix $\mathbf{R}$ are methods to quantify the effects of this overlap.

\section{Acknowledgments}

ASTM Committee E12 on Color and Appearance seeks to develop a new method to evaluate color rendering by lights. This article is intended as research in support of that goal, but is not a standard, nor approved by ASTM International, formerly the American Society for Testing and Materials. Committee members William Thornton and Nick Lena contributed data, and other members have given encouragement and comments. Calculations and graphs were done with a software package called O-Matrix. Reader comments are welcomed, by mail, by email to jime jimworthey . com, or by phone to 1-301-977-3551 in the USA. 


\section{References}

1. James A. Worthey, "Lighting quality and light source size," J. Illum. Eng. Soc. 19(2):142-148 (Summer 1990).

2. James A. Worthey, "Color rendering: a calculation that estimates colorimetric shifts," Color Research and Application, article in press.

3. CIE 13.3-1995, Method of measuring and specifying colour rendering properties of light sources, 3rd edition. This edition contains the same technical recommendations as the $2 \mathrm{nd}$ edition of 1974, but has been updated with regard to spectroradiometric and calculation practice.

4. James A. Worthey, "Opponent-colors approach to color rendering," J. Opt. Soc. Am. 72(1):7482 (1982).

5. William A. Thornton, "Color-discrimination index," J. Opt. Soc. Am. 62(2):191-194.

6. William A. Thornton and E. Chen, "What is visual clarity?" J. Illum. Eng. Soc. 7(2):85-94 (1978).

7. H. Xu, "Color-rendering capacity of illumination," J. Opt. Soc. Am. 73:1709, 1983.

8. H. E. Ives, "The relation between the color of the illuminant and the color of the illuminated object," Trans. Illum. Eng. Soc. 7, 62-72 (1912).

9. James A. Worthey, "Limitations of color constancy," J. Opt. Soc. Am. A2(7):1014-1026 (1985).

10. D. B. Judd, D. L. MacAdam, and G. W. Wyszecki, "Spectral distribution of typical daylight as a function of correlated color temperature," J. Opt. Soc. Am. 54,1031-1040 (1964).

11. Solux data from Philip Bradfield, Tailored Lighting Inc., in 2000.

12. Measurement by the author in 1985 .

13. James A. Worthey, "Global features of color rendering and vision difficulties under fluorescent lights," Ph.D. thesis (Indiana University, Bloomington, Indiana, 1981).

14. John J. McCann, Suzanne P. McKee, and Thomas H. Taylor, "Quantitative studies in retinex theory: a comparison between theoretical predictions and observer responses to the "color mondrian' experiments," Vision Res. 16, 445-458 (1976).

15. Edwin H. Land, "The retinex theory of color vision," Sci. Am. 237(6):108-128 (December 1977).

16. Michael J. Vrhel, Ron Gershon, and Lawrence S. Iwan, "Measurement and analysis of object 
reflectance spectra," Color Res. Appl. 19, 4-9, (1994). These data were obtained in digital form by ftp from server ftp.eos.ncsu.edu in directory /pub/spectra.

17. Jozef Cohen, "Dependency of the spectral reflectance curves of the Munsell color chips," Psychonom. Sci. 1, 369-370 (1964).

18. R. W. G. Hunt, The reproduction of color, 3rd ed. Kings Langley: Fountain Press; 1975; 614 pp; see p. 443.

19. William A. Thornton, “Three-color visual response," J. Opt. Soc. Am. 62(3):457459 (1972).

20. David L. MacAdam, "Photometric relationships between complementary colors," J. Opt. Soc. Am. 28:103-111 (1938).

21. William A. Thornton, "Luminosity and color-rendering capability of white light," J. Opt. Soc. Am. 61(9):1155-1163. William A. Thornton, "A simple picture of matching lights,” J. Illum. Eng. Soc. 8(2):78-85 (1979).

22. P. J. Bouma, "The colour reproduction of incandescent lamps and 'Philiphane Glass,"” Phillips Technical Review 3:47-49 (1938).

23. Data from Nick Lena in 2000.

24. Data from William Thornton in 1980.

25. Jozef B. Cohen and William E. Kappauf, "Metameric color stimuli, fundamental metamers, and Wyszecki's metameric blacks," Am. J. Psych. 95(4):537-564 (1982).

26. Late breaking news: while neodymium lights have been a long-standing niche product, they are now being promoted by a large manufacturer:

http://www.gereveal.com/ .

27. Neodymium glass data courtesy of Corning, Inc., passed along by Daniel Karpen in 2000; related data available on website, http://www.corning.com .

28. Günter W. Wyszecki and W. S. Stiles, Color Science (Wiley, New York, 1967).

29. James A. Worthey, "Calculation of metameric reflectances," Color Res. Appl. 13(2):76-84 (1988). 